\title{
ANG II promotes IGF-IIR expression and cardiomyocyte apoptosis by inhibiting HSF1 via JNK activation and SIRT1 degradation
}

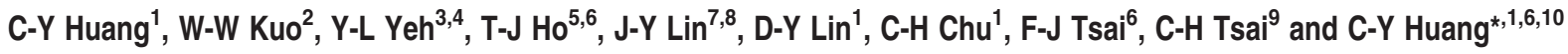

Hypertension-induced cardiac hypertrophy and apoptosis are major characteristics of early-stage heart failure. Our previous studies found that the activation of insulin-like growth factor receptor II (IGF-IIR) signaling was critical for hypertensive angiotensin II (ANG II)-induced cardiomyocyte apoptosis. However, the detailed mechanism by which ANG II regulates IGF-IIR in heart cells remains elusive. In this study, we found that ANG II activated its downstream kinase JNK to increase IGF-IIR expression through the ANG II receptor angiotensin type 1 receptor. JNK activation subsequently led to sirtuin 1 (SIRT1) degradation via the proteasome, thus preventing SIRT1 from deacetylating heat-shock transcription factor 1 (HSF1). The resulting increase in the acetylation of HSF1 impaired its ability to bind to the IGF-IIR promoter region (nt -748 to -585 ). HSF1 protected cardiomyocytes by acting as a repressor of IGF-IIR gene expression, and ANG II diminished this HSF1-mediated repression through enhanced acetylation, thus activating the IGF-IIR apoptosis pathway. Taken together, these results suggest that HSF1 represses IGF-IIR gene expression to protect cardiomyocytes. ANG II activates JNK to degrade SIRT1, resulting in HSF1 acetylation, which induces IGF-IIR expression and eventually results in cardiac hypertrophy and apoptosis. HSF1 could be a valuable target for developing treatments for cardiac diseases in hypertensive patients.

Cell Death and Differentiation (2014) 21, 1262-1274; doi:10.1038/cdd.2014.46; published online 2 May 2014

Apoptosis has been implicated in a wide variety of cardiovascular disorders, including myocardial infarction and heart failure, suggesting that activation of apoptotic pathways contributes to cardiomyocyte loss and subsequently cardiac dysfunction. Previous studies reported that several extracellular molecules, such as insulin-like growth factors (IGFs) and angiotensin II (ANG II), are involved in the development of cardiac hypertrophy and apoptosis. ${ }^{1,2}$ Elevated levels of the vessel contractor protein ANG II are commonly observed in hypertensive patients with cardiovascular diseases and heart failure; these findings suggest that stimulation by ANG II in the heart is associated with an increased rate of myocardial apoptosis. ${ }^{3}$ Previous studies demonstrated that the binding of ANG II to its receptors activates the JNK, ERK and p38 pathways, implying that these downstream effectors may be implicated in the ANG II-induced cardiac cell hypertrophy. ${ }^{4}$ However, the mechanism by which ANG II-induced IGF receptor (IGF-IIR) expression in heart cells leads to apoptosis remains elusive.

The IGF-IIR is a $300-\mathrm{kDa}$ multifunctional type I transmembrane glycoprotein that is involved in lysosomal enzyme trafficking, IGF II clearance and tumor suppression. ${ }^{5,6}$ Several studies have shown fetal overgrowth and neonatal lethality in IGF-IIR-deficient mice as a consequence of major cardiac abnormalities, indicating that IGF-IIR has a vital role in normal cardiac morphogenesis and normal fetal growth. ${ }^{7,8}$ Our previous studies found that the upregulation of the IGF-II and IGF-IIR genes is essential for ANG II-induced cell apoptosis and correlates with the promotion of cardiomyocyte apoptosis in hypertensive rat hearts. ${ }^{9-13}$ However, the detailed mechanisms underlying IGF-IIR gene regulation and the upregulation of IGF-IIR expression by ANG II remain unknown.

In this pioneering study, we first found that deacetylated heat-shock transcription factor 1 (HSF1) suppressed IGF-IIR gene expression. However, ANG II increased IGF-IIR expression by activating the downstream JNK via angiotensin type 1 receptor $\left(A T_{1} R\right)$ to degrade the HSF1 deacetylase sirtuin 1 (SIRT1). SIRT1 degradation then led to HSF1 acetylation, thus preventing HSF1 from binding to the IGF-IIR promoter (nt -748 to -585 ) and repressing IGFIIR expression. This led to an increase in the level of IGF-IIR

\footnotetext{
${ }^{1}$ Graduate Institute of Basic Medical Science, China Medical University, Taichung, Taiwan, ROC; ${ }^{2}$ Department of Biological Science and Technology, China Medical University, Taichung, Taiwan, ROC; ${ }^{3}$ Department of Pathology, Changhua Christian Hospital, Changhua, Taiwan, ROC; ${ }^{4}$ Department of Medical Technology, Jen-Teh Junior College of Medicine, Nursing and Management, Miaoli, Taiwan, ROC; ${ }^{5}$ Chinese Medicine Department, China Medical University Beigang Hospital, Beigang, Taiwan, ROC; ${ }^{6}$ School of Chinese Medicine, China Medical University, Taichung, Taiwan, ROC; ${ }^{7}$ Department of Medical Imaging and Radiological Science, Central Taiwan University of Science and Technology, Taichung, Taiwan, ROC; ${ }^{8}$ Department of Nursing, Central Taiwan University of Science and Technology, Taichung, Taiwan, ROC; ${ }^{9}$ Department of Healthcare Administration, Asia University, Taichung, Taiwan, ROC and ${ }^{10}$ Department of Health and Nutrition Biotechnology, Asia University, Taichung, Taiwan, ROC

*Corresponding author: C-Y Huang, Graduate Institute of Basic Medical Science, China Medical University, Taichung 40402, Taiwan, ROC. Tel: + 886422053366 ext. 3313; Fax: + 8864 22051276; E-mail: cyhuang@ @ail.cmu.edu.tw

Abbreviation: IGF-IIR, insulin-like growth factor receptor II; HSF1, heat-shock transcription factor 1; ANG II, angiotensin II; IGF, insulin-like growth factor; $A T_{1} R$, angiotensin type 1 receptor; SIRT1, sirtuin 1 ; HSE, heat-shock element; RSV, resveratrol; SHR, spontaneously hypertensive rat

Received 13.12.13; revised 07.3.14; accepted 10.3.14; Edited by G Melino; published online 02.5.14
} 
and its translocation to the membrane, resulting in downstream hypertrophy and initiation of the apoptosis signaling pathway in ANG II-stimulated cardiomyocytes and hypertensive hearts.

\section{Results}

ANG II stimulated IGF-IIR gene expression via its receptor $\mathbf{A} T_{1} \mathbf{R}$. Our previous studies demonstrated that ANG II increased IGF-IIR mRNA expression via acetylation of histones $\mathrm{H} 3$ and $\mathrm{H} 4$, suggesting that ANG II regulated IGF-IIR expression in $\mathrm{H} 9 \mathrm{c} 2$ cardiomyoblast cells. ${ }^{14}$ ANG II has been reported to activate downstream signaling via the angiotensin type 1 and type 2 receptors $\left(A T_{1} R\right.$ and $A T_{2} R$, respectively) to elicit various biological responses. ${ }^{1,2}$ As shown in Figure 1a, the knockdown of $A T_{1} R$ reduced the upregulation of IGF-IIR expression by ANG II, whereas the knockdown of $A T_{2} R$ had no obvious influence on IGF-IIR regulation by ANG II. This finding implies that the ANG II-mediated enhancement of IGF-IIR mRNA expression might occur via $A T_{1} R$. We then treated the H9c2 cells with either an $A T_{1} R$ blocker losartan or an $A T_{2} R$ blocker PD123319. Similar to the $A T_{1} R$ knockdown, losartan alleviated the ANG II-mediated induction of IGF-IIR mRNA expression (Figure 1b).

Next, we performed a luciferase reporter assay to measure IGF-IIR promoter activity. The promoter activity of IGF-IIR increased after treatment with ANG II, but there was no significant ANG II-mediated increase in the cells with silenced $A T_{1} R$ (Figure 1c). Similarly, losartan also suppressed the stimulatory effect of ANG II on IGF-IIR expression. The forced expression of Flag-AT ${ }_{1} R$ enhanced IGF-IIR promoter activity, suggesting that ANG II affected IGF-IIR mRNA expression via $\mathrm{AT}_{1} \mathrm{R}$ (Figures $1 \mathrm{c}$ and $\mathrm{d}$ ).

We also measured IGF-IIR protein expression in $\mathrm{H} 9 \mathrm{c} 2$ cells with silenced or blocked $A T_{1} R$. As expected, a reduction in the IGF-IIR protein levels was observed in cells with silenced or blocked $A T_{1} R$ (Figure $1 \mathrm{e}$ ). The overexpression of $A T_{1} R$ significantly enhanced IGF-IIR expression, which was accompanied by increased caspase-3 cleavage (Figure 1f). Our previous studies demonstrated that surface IGF-IIR participated in IGF-IIR-induced cardiac hypertrophy and the apoptosis pathway. We thus measured the levels of membrane IGF-IIR by an enzyme-linked immunosorbent assay (ELISA; Figure 1g). The results showed that the upregulation of membrane IGF-IIR following ANG II exposure was also $A T_{1} R$-dependent. In the absence of $A T_{1} R, A N G$ II only slightly increased the levels of membrane IGF-IIR. Conversely, the overexpression of $A T_{1} R$ stimulated IGF-IIR translocation to the membrane. Taken together, these results indicate that ANG II stimulated the IGF-IIR apoptosis pathway via $A T_{1} R$ in cardiomyocytes.

JNK activation was involved in regulating IGF-IIR expression and membrane translocation. Several studies have reported that $A T_{1} R$ activation triggers the MAPK, p38 and JNK signaling pathways, leading to a variety of cellular outcomes. ${ }^{1,3,4}$ Therefore, we wanted to determine which downstream signaling pathway participated in the regulation of IGF-IIR expression. By kinase inhibitor treatment, we found IGF-IIR expression decreased when PI3K inhibitor (LY294002), MEK inhibitor (U0126), ERK inhibitor (PD98059) or JNK inhibitor (SP600125) - but not p38 inhibitor (SB203580) - was combined with ANG II treatment. The knockdown of these kinases by siRNA similarly showed that PI3K, ERK and JNK were involved in the regulation of IGF-IIR expression, particularly with an ANG II treatment (Figure 2b).

Next, we determined whether the surface accumulation of IGF-IIR was reduced following these kinase inhibitor challenges. As anticipated, the levels of surface IGF-IIR decreased after the addition of the protein kinase inhibitors (Figure 2c). However, only treatment with an MEK inhibitor (U0126) or a JNK inhibitor (SP600125) reduced IGF-IIR promoter activity; the JNK inhibitor was particularly effective (Figure 2d). These results indicate that the different kinases regulated IGF-IIR via distinct mechanisms and that primarily JNK participated in the ANG II-induced upregulation of IGF-IIR gene expression, consistent with our previous studies. ${ }^{10}$

To demonstrate that JNK activation was involved in IGF-IIR gene expression, the JNK activator anisomycin was used to challenge $\mathrm{H} 9 \mathrm{c} 2$ cells. As shown in Figure $3 \mathrm{a}$, the expression of IGF-IIR was gradually enhanced by anisomycin in a dosedependent manner. Moreover, IGF-IIR was more strongly located at the plasma membrane in the ANG II-treated $(16.71 \pm 2.17 \%)$ and anisomycin-treated $(17.21 \pm 3.22 \%)$ cells compared with the control cells $(7.13 \pm 2.13 \%)$ (Figures $3 \mathrm{~b}$ and $\mathrm{c}$ ). The treatment with both ANG II and anisomycin triggered IGF-IIR membrane translocation (23.42 $\pm 6.12 \%$ ); however, this phenomenon was inhibited in JNK inhibitortreated cells $(4.23 \pm 1.53 \%)$. These data thus indicate that JNK activation participated in the upregulation of IGF-IIR expression and membrane translocation and promoted the IGF-IIR apoptosis signaling pathway.

Heat-shock transcription factor 1 repressed IGF-IIR gene expression by binding to the IGF-IIR promoter region (nt $-\mathbf{7 4 8}$ to $\mathbf{- 5 8 5}$ ) under normal conditions. Because ANG II stimulated IGF-IIR gene expression, we constructed IGFIIR promoter deletion mutants to investigate which promoter segment affected IGF-IIR expression (Figure 4a). We found that IGF-IIR promoter activity was much higher in the nt $-748 \sim+11$ region compared with that of the nt $-585 \sim+11$ region, suggesting that the deleted region (nt -748 to -585 ) was necessary for IGF-IIR promoter activity (Figure 4b). Using a transcription factor search website (TFSEARCH), we identified one HSF1 binding site (nt -733 to -703 ) in this part of the IGF-IIR promoter (Figure 4c).

To demonstrate that HSF1 bound to the putative heatshock element (HSE) at the IGF-IIR promoter in vivo, we performed chromatin immunoprecipitation (ChIP) for detecting DNA-protein interactions. As anticipated, the IGF-IIR promoter was detected using an HSF1-specific antibody; however, the interaction was abolished in the ANG II-treated cells, suggesting that HSF1 may be a negative regulator of IGF-IIR expression in H9c2 cells (Figure 4d). Therefore, we silenced HSF1 using siRNA and examined the effect on IGF-IIR expression. A luciferase assay showed that the 

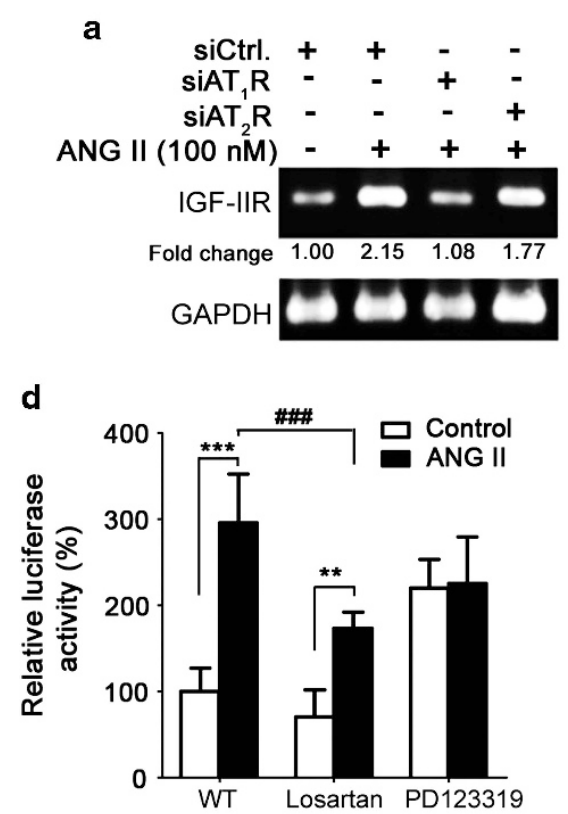

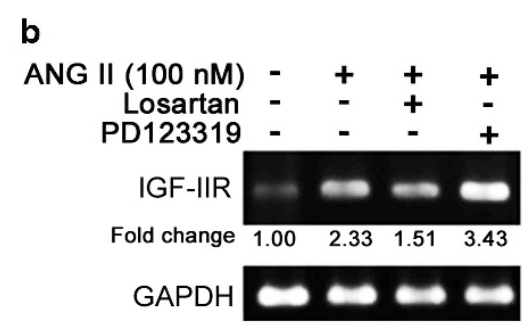

e

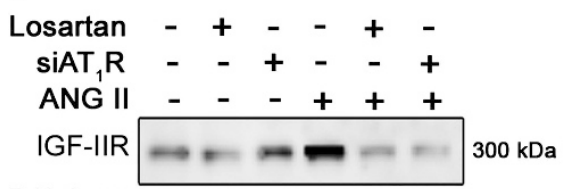

Fold change $\begin{array}{lllllll}1.00 & 0.78 & 1.16 & 1.91 & 0.65 & 0.55\end{array}$

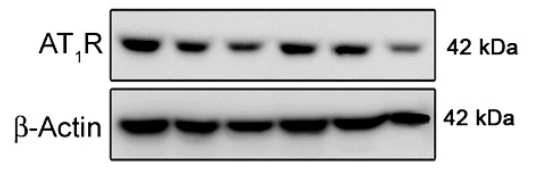

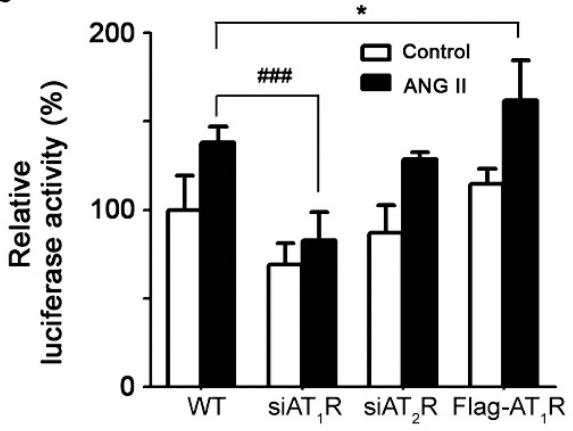

f
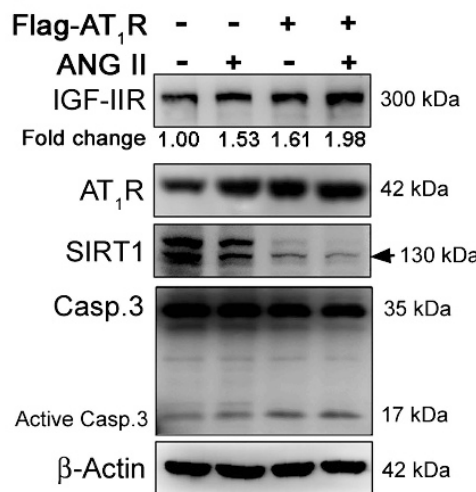

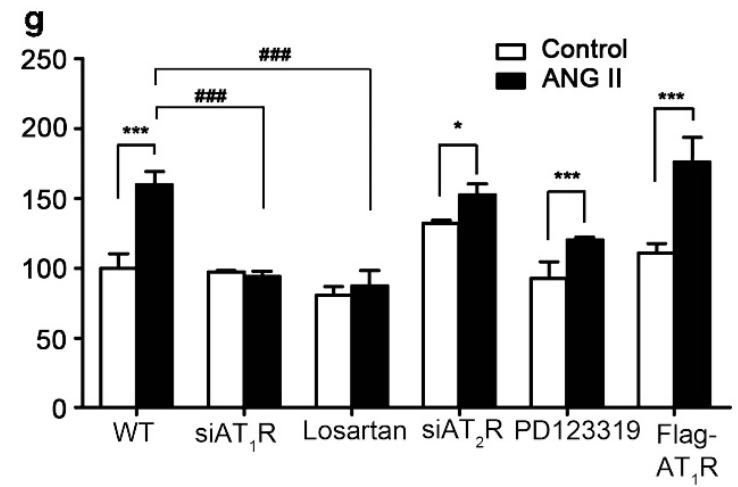

Figure 1 ANG II stimulated IGF-IIR expression to induce apoptosis through the $A T_{1} R$. (a) H9c2 cells were silenced with the $A T_{1} R$ and $A T_{2} R$ small interfering RNAs (siRNAs) $(10 \mathrm{nM})$ for $24 \mathrm{~h}$. Then, the cells were treated with ANG II (100 nM) for $24 \mathrm{~h}$. The expression of the IGF-IIR mRNA was measured using RT-PCR analysis. (b) H9c2 cells were treated with the $\mathrm{AT}_{1} \mathrm{R}$ blocker losartan $(1 \mu \mathrm{M})$ and the $\mathrm{AT}_{2} \mathrm{R}$ blocker PD123319 $(1 \mu \mathrm{M})$ with ANG II $(100 \mathrm{nM})$ for $24 \mathrm{~h}$. The expression of the IGF-IIR mRNA was examined by RT-PCR analysis. (c) H9c2 cells were transfected with pGL4-IGF-IIR (nt $-1249 \sim+11)$ together with an siRNA or overexpression plasmid for $24 \mathrm{~h}$. The cells were then treated with ANG II $(100 \mathrm{nM})$ and assayed for luciferase activity. The results are shown as the means \pm S.D. of three independent experiments. ${ }^{*} P<0.05$, represents a significant increase compared with the untreated control. ${ }^{\# \#} P<0.001$, represents a significant decrease compared with the untreated control. (d) H9c2 cells were treated with losartan or PD123319 with ANG II for $24 \mathrm{~h}$ and then assayed for luciferase activity. The results are shown as the means \pm S.D. of three independent experiments. ${ }^{* *} P<0.01$, represents a significant increase compared with the untreated control. ${ }^{* *} P<0.001$, represents a significant increase compared with the untreated control. ${ }^{\# \#} P<0.001$, represents a significant decrease compared with the untreated control. (e) H9c2 cells were transfected with the AT $R$ siRNA for $24 \mathrm{~h}$ and then treated with ANG II (100 nM) for $24 \mathrm{~h}$ or co-treated with the $A T_{1} R$ blocker losartan and ANG II for $24 \mathrm{~h}$. IGF-IIR and AT ${ }_{1}$ R levels were measured by immunoblotting. (f) H9c2 cells were transfected with Flag-AT $R$ R for $24 \mathrm{~h}$ and then treated with ANG II (100 nM). The levels of IGF-IIR, caspase-3 and $\mathrm{AT}_{1} \mathrm{R}$ were measured by immunoblotting. (g) H9c2 cells were transfected with siAT $R$, siAT $2 R$ and Flag-AT $R$ or treated with Losartan ( $A T_{1} R$ blocker) and PD123319 ( $A T_{2} R$ blocker) for $24 \mathrm{~h}$, and then challenged with $A N G$ II for $24 \mathrm{~h}$. The membrane IGF-IIR was detected by ELISA. ${ }^{*} P<0.5$, represents a significant increase compared with the untreated control. ${ }^{* * \star} P<0.01$, represents a significant increase compared with the untreated control. ${ }^{\# \#} P<0.001$, represents a significant decrease compared with the untreated control. All the results are representative of three independent experiments

knockdown of HSF1 substantially elevated IGF-IIR promoter activity under normal conditions and slightly increased the activity in ANG II-treated cells, suggesting that HSF1 might negatively regulate IGF-IIR expression (Figure 4e). We then used the HSF1 siRNA or an inhibitor (triptolide) for treating cells and detected elevated levels of IGF-IIR in the HSF1deficient cells (Figure 4f). The surface levels of IGF-IIR also increased in the HSF1-silenced cells, but decreased in the HSF1-overexpressing cells. Taken together, these results indicate that HSF1 acts as a transcription repressor to inhibit IGF-IIR expression in cardiomyocytes.

ANG II influenced the HSF1-mediated suppression of IGF-IIR via posttranscriptional modifications. Previous studies demonstrated that posttranslational modifications of HSF1, including phosphorylation and acetylation, can modulate its function by suppressing its transcriptional activity and DNA-binding activity. ${ }^{15,16}$ The phosphorylation 
a

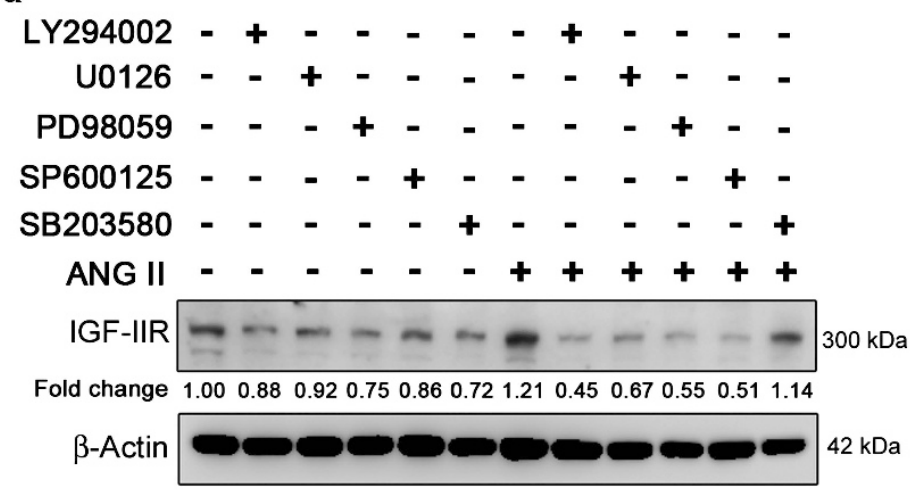

b

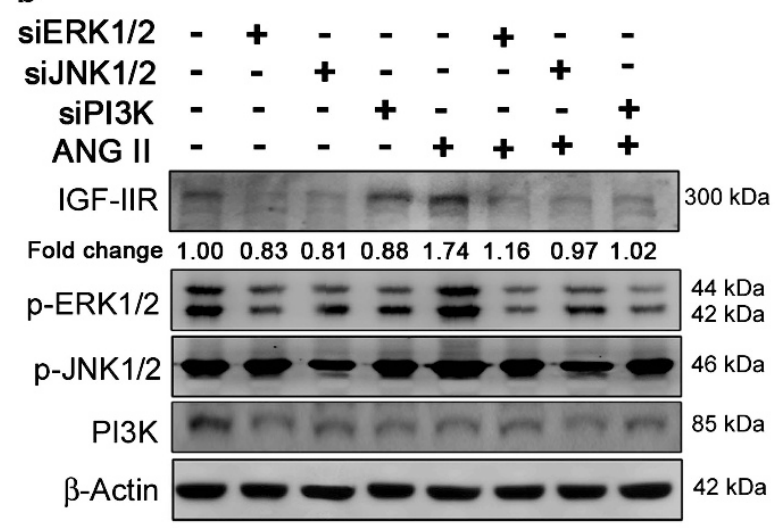

c

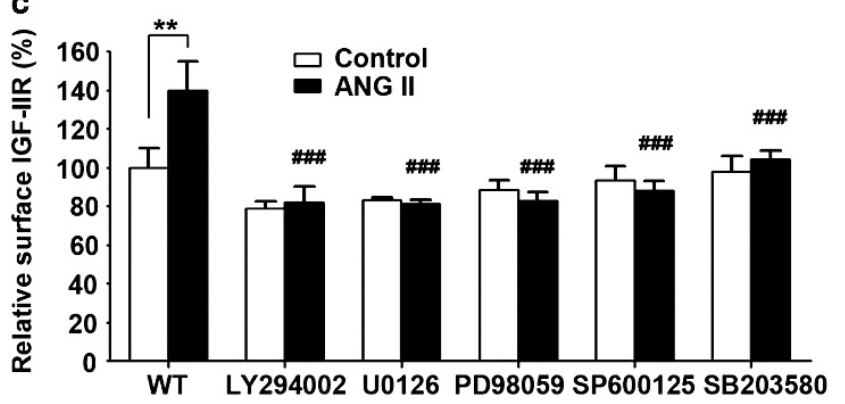

d

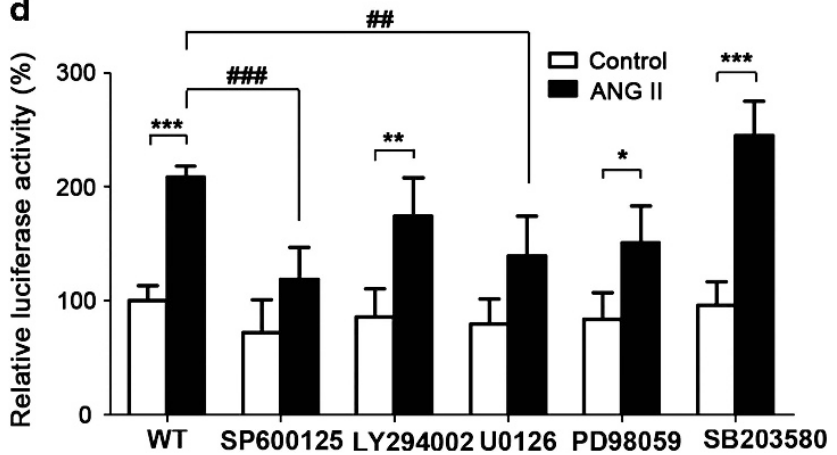

Figure 2 ANG II enhanced IGF-IIR expression via c-Jun N-terminal kinase (JNK), extracellular-signal-regulated kinase (ERK) and phosphatidylinositol 3-kinase (PI3K). (a) H9c2 cells were co-treated with a PI3K inhibitor (LY294002, $10 \mu \mathrm{M}$ ), MEK inhibitor (U0126, 30 $\mu \mathrm{M}$ ), ERK inhibitor (PD98059, $10 \mu \mathrm{M}$ ), JNK inhibitor (SP600125, $20 \mu \mathrm{M}$ ) or p38 inhibitor (SB203580, $10 \mu \mathrm{M}$ ) together with ANG II (100 nM). IGF-IIR expression was measured by immunoblotting. (b) H9c2 cells were treated with small interfering RNAs (siRNAs) (10 nM) for PI3K, ERK and JNK individually. After $24 \mathrm{~h}$, the cells were exposed to ANG II (100 nM). The levels of IGF-IIR, PI3K, phosphorylated (p)-ERK and p-JNK1/2 were examined by immunoblotting. (c) The membrane IGF-IIR of H9c2 cells was detected by ELISA following treatment with kinase inhibitors and ANG II. ${ }^{* *} P<0.01$, represents a significant increase compared with the untreated control. ${ }^{\# \#} P<0.001$, represents a significant decrease compared with the untreated control. (d) H9c2 cells transfected with pGL4-IGF-IIR (nt -1249 to +11 ) for $24 \mathrm{~h}$ and then treated with a distinct kinase inhibitor and ANG II for $24 \mathrm{~h}$. The cells were assayed for luciferase activity. The results are shown as the means \pm S.D. of three independent experiments. ${ }^{*} P<0.05$, represents a significant increase compared with the untreated control. ${ }^{* *} P<0.01$, represents significant increase comparison with untreated control. ${ }^{* \star} P<0.001$, represents a significant increase compared with the untreated control. ${ }^{\#} P<0.01$, represents a significant decrease compared with the untreated control. ${ }^{\# \#} P<0.001$, represents a significant decrease compared with the untreated control. The results are representative of three independent experiments

of HSF1 has been shown to suppress both its transcriptional activity $^{17-20}$ and its DNA-binding activity, ${ }^{21}$ and HSF1 acetylation inhibited the DNA-binding activity. ${ }^{22,23}$ Because HSF1 lost its ability to bind the IGF-IIR promoter region following ANG II treatment, we thought that HSF1 might be phosphorylated or acetylated to inhibit its DNA-binding ability. Using subcellular fractionation, we found that the nuclear HSF1 was reduced, whereas the cytosolic HSF1 accumulated and showed phosphorylation at serine 303 (Figure 5a). Moreover, we observed that the decrease in the levels of nuclear HSF1 was dose-dependent. As shown in Figure 5b, the percentage of nuclear HSF1 decreased from $92.32 \%$ (control cells) to $65.31 \%$ (200 nM ANG II) (Figures 5b and c).

To determine whether HSF1 acetylation was also involved in regulating IGF-IIR expression, we examined the acetylation level of HSF1 under ANG II treatment. Using forced expression of HSF1 and immunoprecipitation, we found that ANG II enhanced the acetylation level of HSF1 (Figure 5d). Likewise, immunoprecipitation with antibodies specific to endogenous HSF1 precipitated the acetylated HSF1 (as detected by antiacetyl lysine antibodies), and vice versa (Figure 5e), suggesting that ANG II induced HSF1 acetylation.

SIRT1 has been shown to serve as an HSF1 deacetylase to regulate its DNA-binding activity by deacetylating the HSF1 Lys80 residue. ${ }^{15,22,23}$ Therefore, SIRT1 expression was measured during ANG II treatment. SIRT1 expression was reduced in ANG II-treated $\mathrm{H} 9 \mathrm{c} 2$ cells compared with that in control cells, implying that ANG II might downregulate SIRT1 expression to induce acetylation of HSF1, thus further activating IGF-IIR expression (Figure 5f). Collectively, these results indicate that ANG II regulated the HSF1-mediated repression of IGF-IIR through posttranslational modifications. The phosphorylated HSF1 that accumulates in the cytoplasm and the acetylated HSF1 are inactive, resulting in activation of the IGF-IIR apoptosis pathway.

The deacetylase SIRT1 negatively regulated IGF-IIR expression by deacetylating HSF1. We next examined whether SIRT1 regulated IGF-IIR expression. Western blot results showed that the knockdown of SIRT1 upregulated IGF-IIR expression (Figure 6a). To determine whether SIRT1 
a

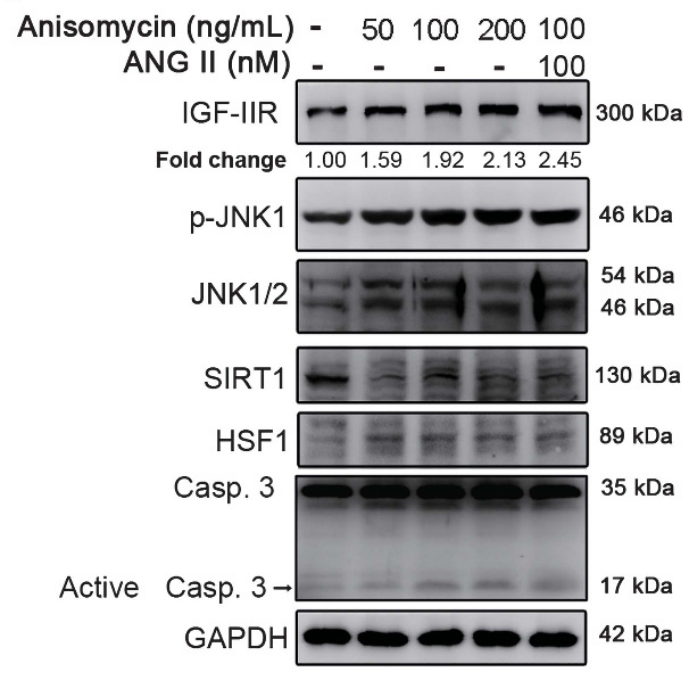

c

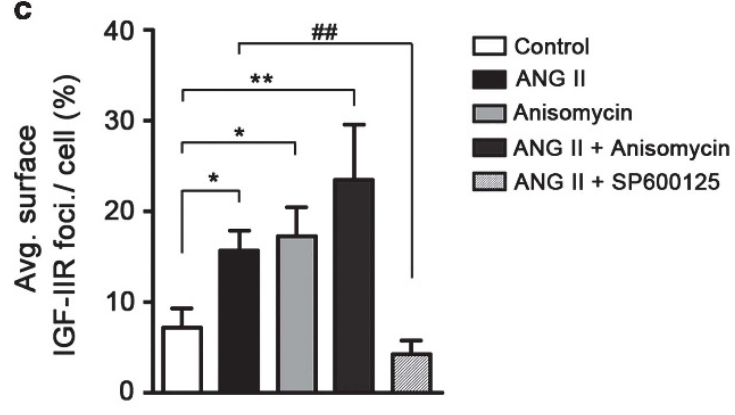

b
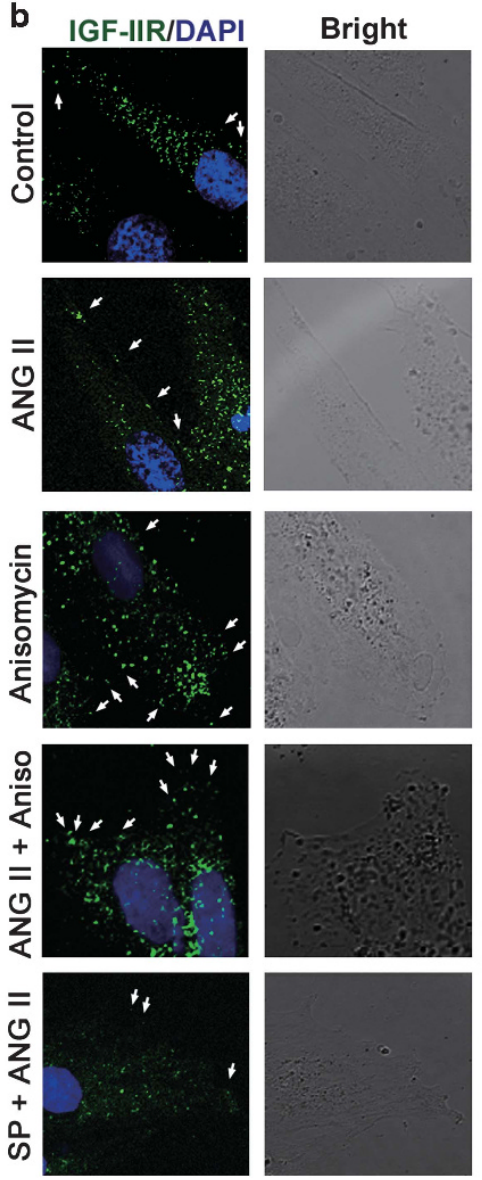

Merge
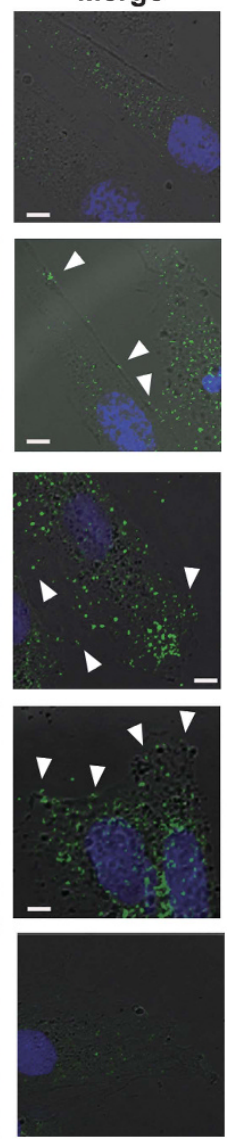

Figure 3 c-Jun N-terminal kinase (JNK) activation upregulated the expression of IGF-IIR, which translocated to the membrane. (a) H9c2 cells were treated with increasing concentrations of the JNK activator anisomycin $(0,50,100$ and $200 \mathrm{nM})$ combined with ANG II. The lysates were analyzed by immunoblotting with IGF-IIR, JNK and caspase-3 antibodies. (b and c) After treatment with anisomycin (JNK activator), SP600125 (JNK inhibitor) and ANG II for $24 \mathrm{~h}$, the cells were fixed with $4 \%$ paraformaldehyde. Immunofluorescence was measured using anti-IGF-IIR antibodies. White arrows indicate the IGF-IIR protein. ${ }^{*} P<0.05$ represents a significant increase compared with untreated control. ${ }^{* *} P<0.01$ represents a significant increase in comparison with untreated control. ${ }^{\# \#} P<0.01$ represents a significant decrease of membrane IGF-IIR in comparison with ANG II-treated cells. All the blots and micrographs are representative of two to three sets of separate experiments

might regulate IGF-IIR gene expression at the transcriptional level, we measured the abundance of the IGF-II mRNA by reverse transcription-polymerase chain reaction (RT-PCR). The results revealed that the silencing of SIRT1 increased IGF-IIR mRNA expression, suggesting that SIRT1 might negatively regulate IGF-IIR to prevent cardiomyocyte apoptosis.

To determine whether the effect of SIRT1 on IGF-IIR expression involved its deacetylase activity, we overexpressed SIRT1-wt and SIRT1-H363Y, a deacetylase-defective mutant, and examined IGF-IIR expression during an ANG II treatment. We found that the SIRT1 activator resveratrol (RSV) attenuated the effect of ANG II on IGF-IIR expression (Figure $6 \mathrm{~b}$ and Supplementary Figure 1A). The overexpression of SIRT1-wt consistently reduced IGF-IIR expression. However, this phenomenon was not observed in SIRT1 H363Y-transfected cells, indicating that the deacetylase activity of SIRT1 is necessary for regulating IGF-IIR expression. Moreover, the overexpressed SIRT1 alleviated the ANG II-induced HSF1 acetylation and IGF-IIR expression, suggesting that SIRT1 negatively regulated IGF-IIR expression by modulating HSF1 acetylation (Figure 6c). Likewise, we found that treatment with the SIRT1 inhibitor nicotinamide (NAM) increased IGF-IIR expression (Figure 6d). However, this effect was eliminated by overexpressing HSF1 (Figure 6d). Altogether, these results show that ANG II degraded SIRT1 to increase HSF1 acetylation, which reduced the ability of HSF1 to downregulate IGF-IIR.

Several studies have shown that JNK activation leads to SIRT1 degradation via the proteasome. ${ }^{24,25}$ Therefore, we hypothesized that ANG II activated the JNK signaling to degrade SIRT1 and further increased HSF1 acetylation. We examined the influence of JNK inhibitor on HSF1 acetylation. As anticipated, the SIRT1 inhibitor NAM enhanced ANG IIinduced HSF1 acetylation; by contrast, the JNK inhibitor attenuated these effects (Figure 6e). We also investigated whether SIRT1 degradation occurs via the ubiquitinproteasome system (Figure 6f). Treatment with the proteasome inhibitor MG132 inhibited the ANG II- or anisomycin-induced SIRT1 degradation, implying that SIRT1 degradation is proteasome-dependent. To test this hypothesis, we immunoprecipitated SIRT1 to determine whether ANG II ubiquitinylated SIRT1. As shown in Figure $6 \mathrm{~g}$, SIRT1 was slightly ubiquitinylated under basal 
a
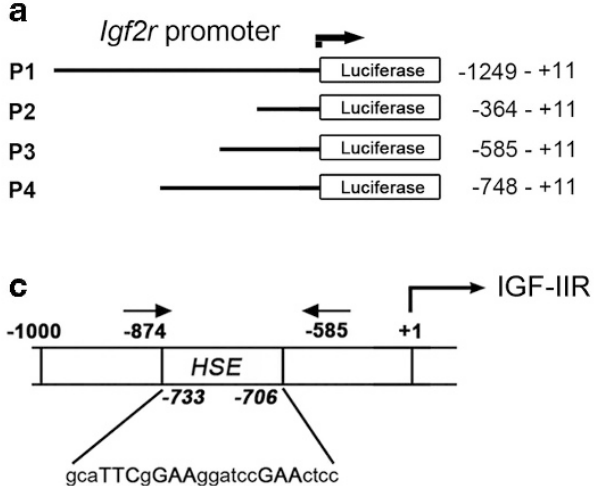

b

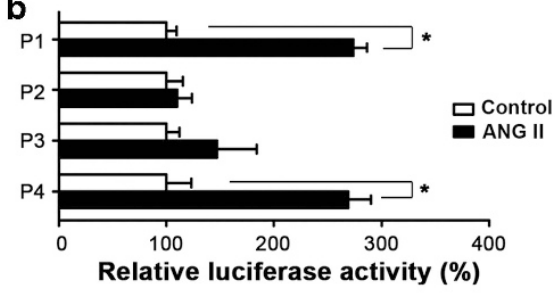

d

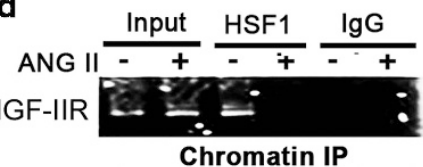

IGF-IIR promoter (nt -748 -585)

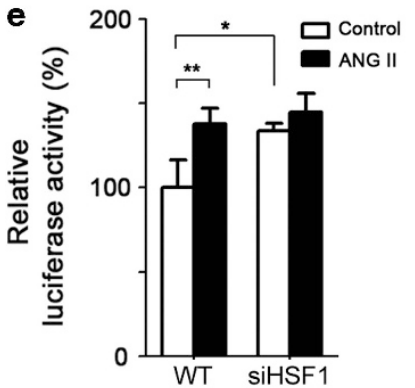

f
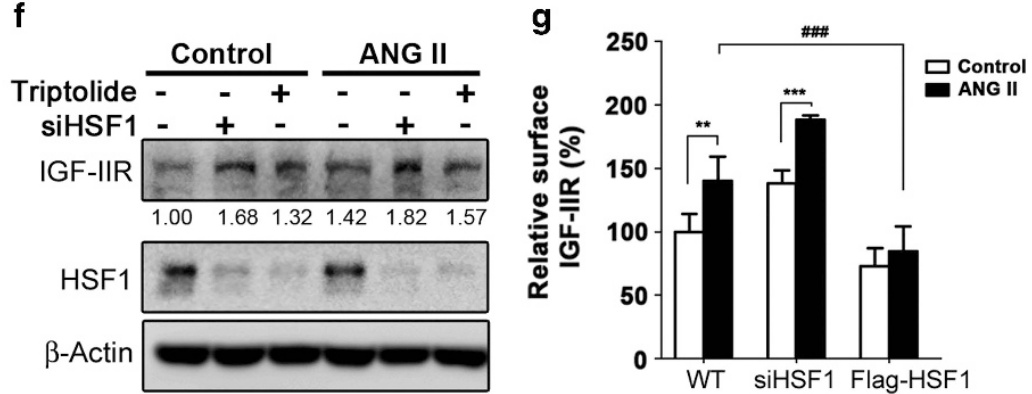

Figure 4 HSF1 suppressed IGF-IIR expression by binding to its promoter region (nt -748 to -585 ). (a) Schematic diagram of the IGF-IIR promoter deletion mutations. (b) H9c2 cells were transfected with different IGF-IIR luciferase constructs for $24 \mathrm{~h}$ and then treated with ANG II for $24 \mathrm{~h}$. Cell lysates were assayed for luciferase activity. ${ }^{*} P<0.05$ represents a significant increase in comparison with untreated control. (c) Sequence analysis and transcription factor site prediction identified one putative HSF1 binding element at the IGF-IIR promoter (nt - 733 to - 706). (d) After treatment with ANG II, the H9c2 cells were lysed and analyzed by ChIP. HSF1 binding to the IGF-IIR promoter was quantified using PCR. (e) HSF1 was silenced with small interfering RNA (siRNA) (10 nM) and transfected with pGL4-IGF-IIR (nt - 1249 + 11) for 24h, followed by exposure to ANG II (100 nM) for $24 \mathrm{~h}$. The lysates were analyzed for luciferase activity. ${ }^{*} P<0.05$ represents a significant increase in comparison with untreated control. ${ }^{* *} P<0.01$ represents a significant increase in comparison with untreated control. (f) After treatment with the HSF1 inhibitor triptolide (1 $\left.\mu \mathrm{M}\right)$ and the HSF1 siRNA $(10 \mathrm{nM})$ for $24 \mathrm{~h}$, H9c2 cells were challenged with ANG II (100 nM). IGF-IIR and HSF1 levels were measured by immunoblotting. (g) H9c2 cells were silenced with the HSF1 siRNA $(10 \mathrm{nM})$ or the HSF1 inhibitor triptolide $(1 \mu \mathrm{M})$ for $24 \mathrm{~h}$ and then challenged with ANG II (100 nM) for $24 \mathrm{~h}$. The membrane levels of IGF-IIR were analyzed by ELISA. ${ }^{* \star} P<0.01$ represents a significant increase in comparison with untreated control. ${ }^{* \star *} P<0.001$ represents a significant increase in comparison with untreated control. $\#$ \#\# $P<0.001$ represents a significant decrease in comparison with untreated control. All the blots are representative of two to three sets of independent experiments

conditions, but ANG II increased its ubiquitinylation level. Taken together, these results demonstrate that SIRT1 negatively regulated IGF-IIR expression by deacetylating HSF1; however, ANG II activated JNK to degrade SIRT1 and increase HSF1 acetylation, thus enhancing IGF-IIR expression.

JNK enhances SIRT1 degradation, causing HSF1 acetylation and activation of IGF-IIR-induced hypertrophy and apoptosis in rat primary cardiomyocytes and animal models.

To further demonstrate that ANG II upregulated IGF-IIR via JNK activation and SIRT1 degradation in H9c2 cells, we used the JNK activator anisomycin, the SIRT1 activator RSV and the SIRT1 inhibitor NAM and examined their effect on ANG IIinduced IGF-IIR expression. As shown in Figure 7a, the ANG II and anisomycin treatments resulted in JNK activation. These treatments led to SIRT1 degradation and upregulation of IGF-IIR, which activated its downstream protein $\mathrm{G} \alpha \mathrm{q}$ to cleave caspase-3 and increase the hypertrophy marker BNP. Interestingly, the SIRT1 inhibitor NAM enhanced the effect of ANG II on the expression of IGF-IIR, G $\alpha$, caspase-3 and BNP. The SIRT1 activator RSV neutralized the effect of ANG II, suggesting that SIRT1 is the downstream effector of JNK. Moreover, we observed that JNK was activated to degrade SIRT1, resulting in HSF $1^{\text {Ser303 }}$ phosphorylation and IGF-IIR upregulation in a dose- and time-dependent manner under the ANG II challenge (Supplementary Figures $1 \mathrm{~A}$ and $\mathrm{B}$ ). The SIRT1 activator RSV alleviated the effect of ANG II, thus rescuing SIRT1 expression and reducing IGF-IIR expression (Supplementary Figure 1C).

The same phenomenon was observed in neonatal rat ventricular myocytes (NRVMs) (Figure 7b). We isolated primary NRVMs and transfected them with Flag-HSF1 and the HSF1 siRNA. Following transfection, we challenged the cells with the JNK activator, JNK inhibitor and SIRT1 inhibitor. Anisomycin enhanced the effects of ANG II on IGF-IIR upregulation, SIRT1 degradation and caspase-3 cleavage. By contrast, the overexpression of HSF1 weakened the influence of both ANG II and anisomycin on IGF-IIR expression and caspase-3 activation. Similar results were observed under SIRT1 inhibitor treatment, but SP600125 diminished the effects. These results indicate that ANG II upregulated IGF-IIR through JNK activation, which led to SIRT1 degradation and a resulting increase in HSF1 acetylation. The enhanced expression of IGF-IIR eventually activated the downstream hypertrophy and apoptosis pathways.

To further confirm our hypothesis in vivo, we assayed protein expression in the heart tissue of spontaneously 
a

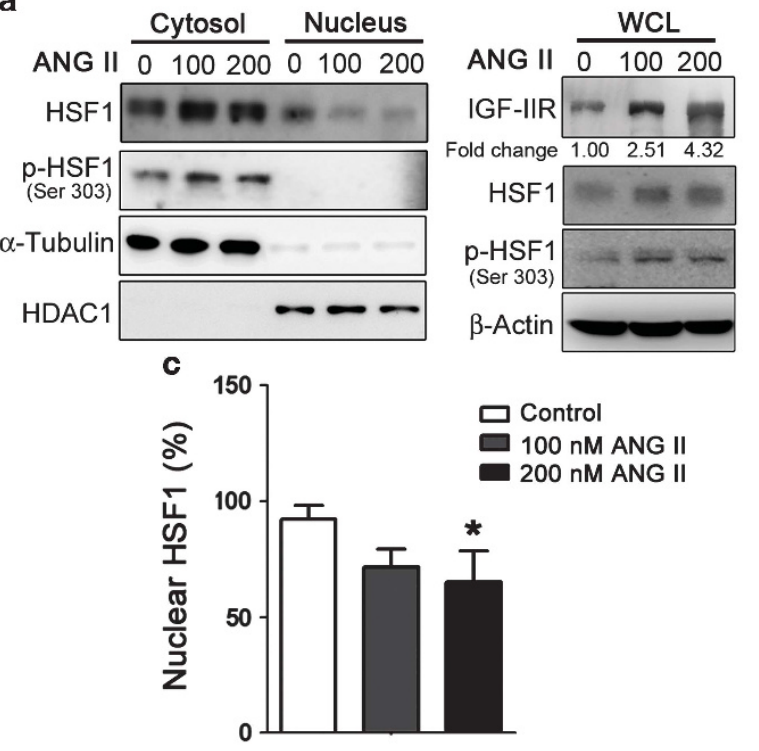

b
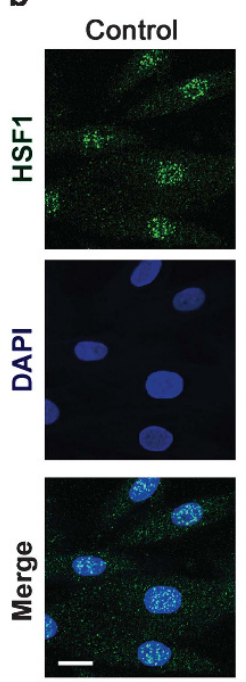
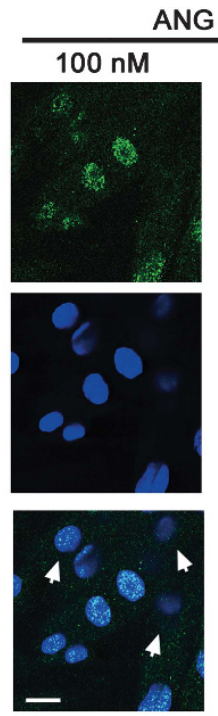

NG II
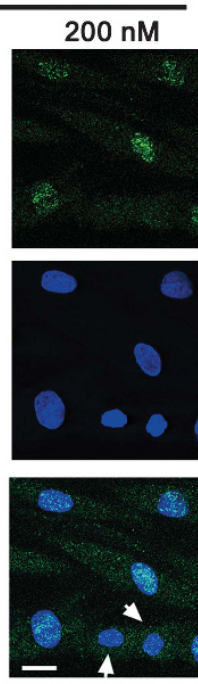

d

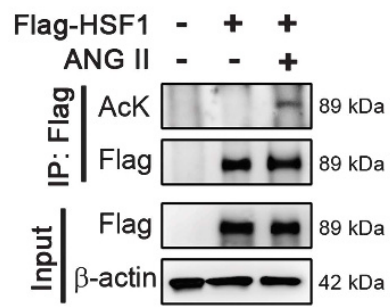

e

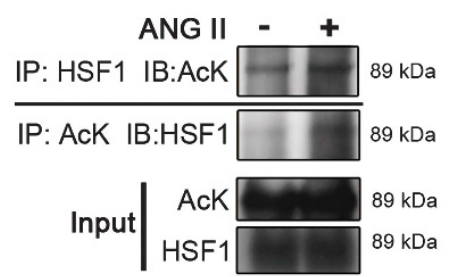

f

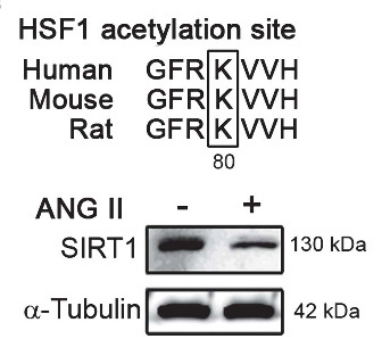

Figure 5 The ability of HSF1 to repress IGF-IIR was reduced by ANG II via posttranslational modification. (a) H9c2 cells were treated with ANG II, and the cell lysates were fractionated into cytosolic and nuclear proteins. HSF1 (both the active and the Ser303-phosphorylated inactive form) were analyzed by immunoblotting. $\alpha$-Tubulin and histone deacetylase (HDAC) served as the cytosolic and nuclear loading controls. (b and c) H9c2 cells were cultured on slides and treated with ANG II for $24 \mathrm{~h}$. Immunofluorescence analysis was performed with the anti-HSF1 antibody and Alexa Fluor 488. The nuclear compartment was stained with 4',6-diamidino-2-phenylindole (DAPI), and the cells were visualized under a confocal microscope. ${ }^{*} P<0.05$, represents a significant increase compared with the untreated control. (d) H9c2 cells were transfected with Flag-HSF1 for $24 \mathrm{~h}$ and treated with ANG II for $24 \mathrm{~h}$. Then, $500 \mu \mathrm{g}$ of cell lysate was immunoprecipitated with anti-Flag antibodies and detected with anti-acetyl-lysine antibodies by immunoblotting. (e) H9c2 cells were treated with ANG II for $24 \mathrm{~h}$. Then, $500 \mu \mathrm{g}$ of cell lysate was immunoprecipitated with anti-HSF1 and anti-acetyl antibodies and analyzed by immunoblotting. (f) H9c2 cells were treated with ANG II for $24 \mathrm{~h}$. The HSF1 acetylated residue, lysine 80 , is conserved in human, mouse and rat. SIRT1 levels were assayed by immunoblotting

hypertensive rats (SHRs) and SHR challenged with ANG II receptor blockers (ARBs). As with the ANG II treatment, we observed JNK activation, a reduction in SIRT1 and HSF1 and upregulation of IGF-IIR, BNP and activated caspase-3 in SHR heart tissue. Upon treatment with an antihypertension drug (irbesartan), the SIRT1 and HSF1 levels were recovered. Consequently, the IGF-IIR-induced cardiac hypertrophy and apoptosis were ameliorated (Figures 7c and d).

\section{Discussion}

In this study, we demonstrated that ANG II increased IGF-IIR expression via an elaborate mechanism, which involves activation of the downstream kinase JNK $1 / 2$ through the $A T_{1} R$ to degrade the deacetylase SIRT1 via the proteasome. SIRT1 degradation then resulted in enhanced acetylation of HSF1, which diminished its ability to suppress IGF-IIR gene expression. IGF-IIR, whose expression was elevated, translocated to the plasma membrane and contributed to the progression of pathological hypertrophy and apoptosis (Figure 8). These findings provide a well-characterized mechanism that may direct a therapeutic strategy for regulating hypertension-induced heart failure through the JNK1/2-SIRT1-HSF1-IGF-IIR pathway.

Several studies have shown that the loss of IGF-IIR results in fetal overgrowth, neonatal lethality and elevated levels of circulating IGF-II. ${ }^{7}$ This embryonic death appears to be the consequence of major cardiac abnormalities, suggesting that IGF-IIR has an essential role in fetal growth and heart development. ${ }^{7,8,26,27}$ Our previous studies clearly demonstrated that the upregulation of IGF-II/IGF-IIR was involved in ANG II-induced cardiomyocyte apoptosis and was correlated with promoting cardiomyocyte apoptosis in hypertensive rat hearts. These results suggested that the apoptotic effect of ANG II might be caused by activation of the IGF-IIR signaling cascade. ${ }^{10,28}$ In this study, we have demonstrated that JNK1/2 activation positively regulated IGF-IIR gene expression. IGF-IIR, whose expression was enhanced, translocated 
a

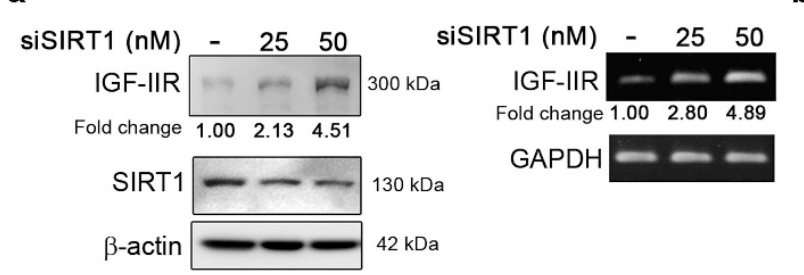

c
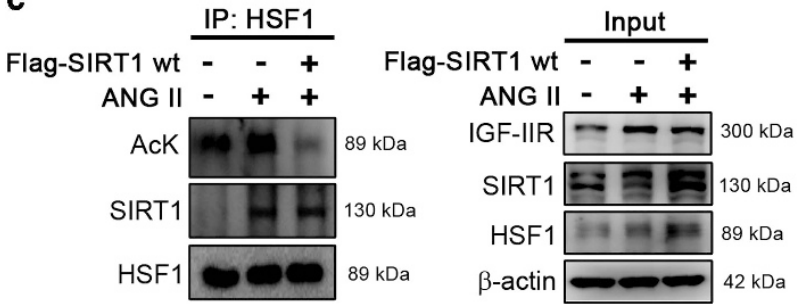

e

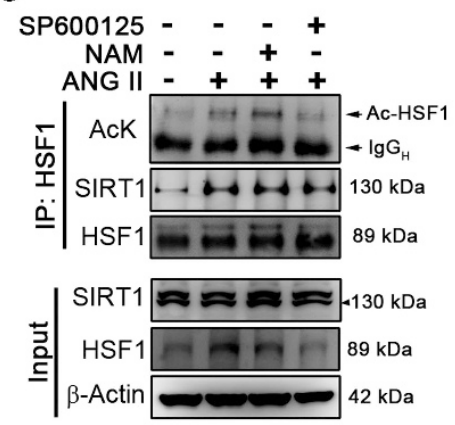

b

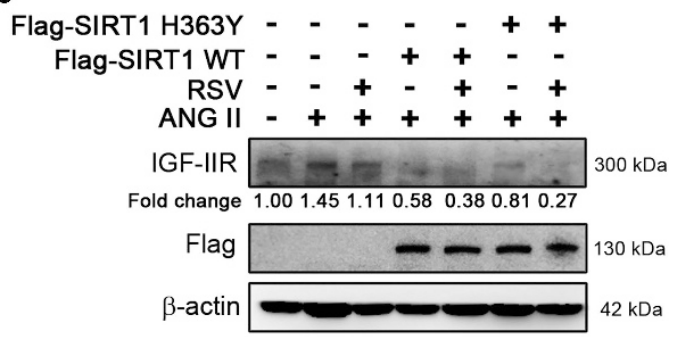

f

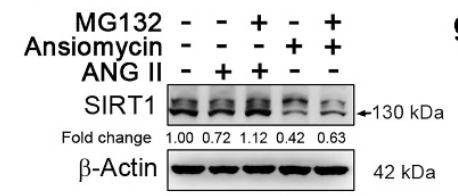

d

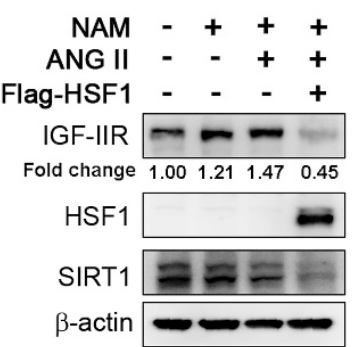

g

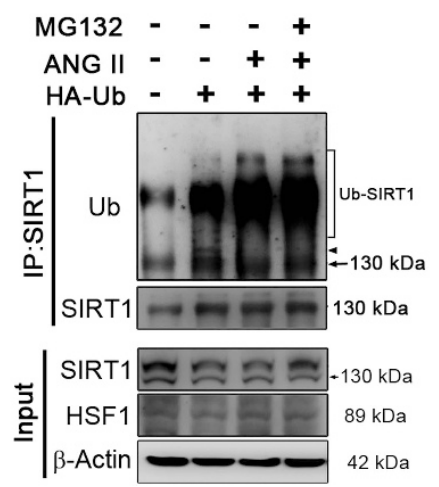

Figure 6 JNK induced IGF-IIR expression by degrading SIRT1 via the proteasome to impair HSF1 deacetylation. (a) H9c2 cells were silenced with the SIRT1 small interfering RNA (siRNA) for $24 \mathrm{~h}$ and analyzed by RT-PCR and immunoblotting. (b) After transfection with SIRT1-wt or SIRT1-H363Y (deacetylase defective mutant) for $24 \mathrm{~h}$, H9c2 cardiomyoblast cells were treated with ANG II ( $100 \mathrm{nM}$ ) alone or combined with the SIRT1 activator RSV ( $50 \mathrm{mM})$ for $24 \mathrm{~h}$. The expression of IGF-IIR was assayed by immunoblotting. (c) H9c2 cells were transfected with SIRT1-wt (wild-type) and treated with ANG II. The lysates were analyzed by immunoprecipitation and immunoblotting. (d) H9c2 cells were transfected with Flag-HSF1 for $24 \mathrm{~h}$ and treated with ANG II alone or combined with the SIRT1 inhibitor NAM (10 mM) for $24 \mathrm{~h}$. The expression of IGF-IIR and SIRT1 was examined by immunoblotting. (e) H9c2 cells were treated with ANG II alone or combined with NAM (10 mM) and SP600125 (20 $\mu$ M) and then analyzed by immunoprecipitation and immunoblotting. The acetylation of HSF1 was detected using an anti-acetyl-lysine antibody. (f) H9c2 cells were treated with ANG II or anisomycin for $15 \mathrm{~h}$, followed by the addition of the proteasome inhibitor MG132 $(10 \mathrm{mM})$ for $9 \mathrm{~h}$. The expression of SIRT1 was assayed by immunoblotting. (g) H9c2 cells were transfected with hemagglutinin (HA)-ubiquitin for $24 \mathrm{~h}$, and then ANG II and MG132 were added. The lysates were immunoprecipitated with specific SIRT1 antibodies and analyzed by immunoblotting

to the plasma membrane and sensitized IGF-II to induce apoptosis. These findings support our previous studies. ${ }^{10,14,29}$ We also observed that other kinases downstream of $A T_{1} R$ were involved in IGF-IIR expression. However, these kinases may have influenced IGF-IIR expression via different mechanisms, such as protein stability and degradation. The detailed mechanisms should be elucidated in future studies.

SIRT1 is considered to be a cytoprotective factor, which is involved in the pathogenesis of obesity, diabetes and aging. Previous studies have indicated that the loss of SIRT1 led to dilated cardiomyopathy and mitochondrial abnormality in the adult hearts of SIRT1-deficient mice. ${ }^{30}$ Recently, several reports demonstrated that ANG II reduces SIRT1 expression, whereas the ANG II-induced cell hypertrophy is diminished by the activation of SIRT1. ${ }^{31,32}$ Moreover, the administration of RSV, a specific SIRT1 activator, suppressed cardiac hypertrophy and restored cardiac function, suggesting that SIRT1 is essential for cardiomyocyte development. ${ }^{33,34}$ Previous studies showed that JNK activation influences SIRT1 protein stability, ${ }^{24,25}$ consistent with our observations. We found that a JNK1/2 activator (anisomycin) promoted SIRT1 degradation via the ubiquitinproteasome system. A proteasome inhibitor alleviated the JNK1/2-induced SIRT1 degradation. Therefore, our results demonstrate that ANG II reduced SIRT1 protein expression via JNK activation.

HSF1 is a transcription factor associated with cellular responses to divergent pathophysiological stresses, such as myocardial ischemia/reperfusion. ${ }^{35-37}$ In unstressed cells, HSF1 is present in both the cytoplasm and the nucleus. In response to stress stimuli, HSF1 accumulates within the 
a
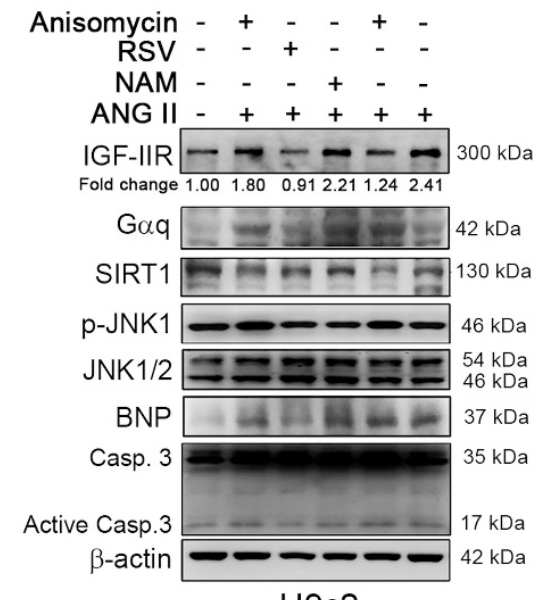

$\mathrm{H} 9 \mathrm{c} 2$

c

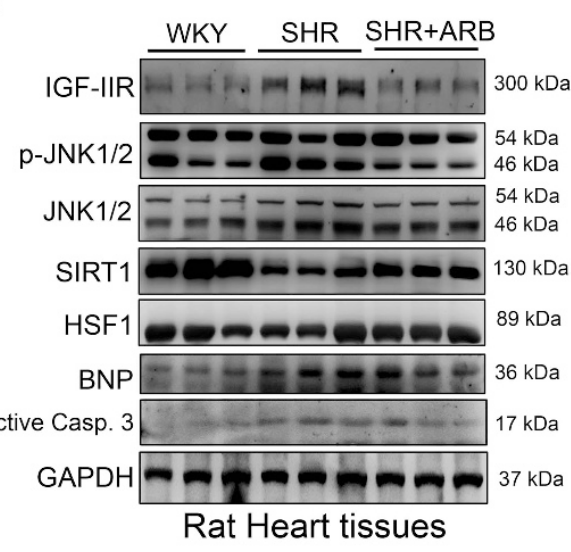

b

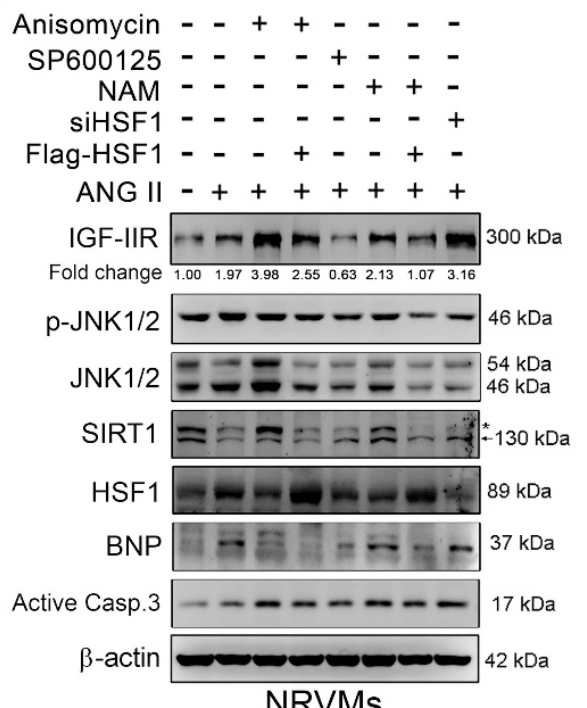

d
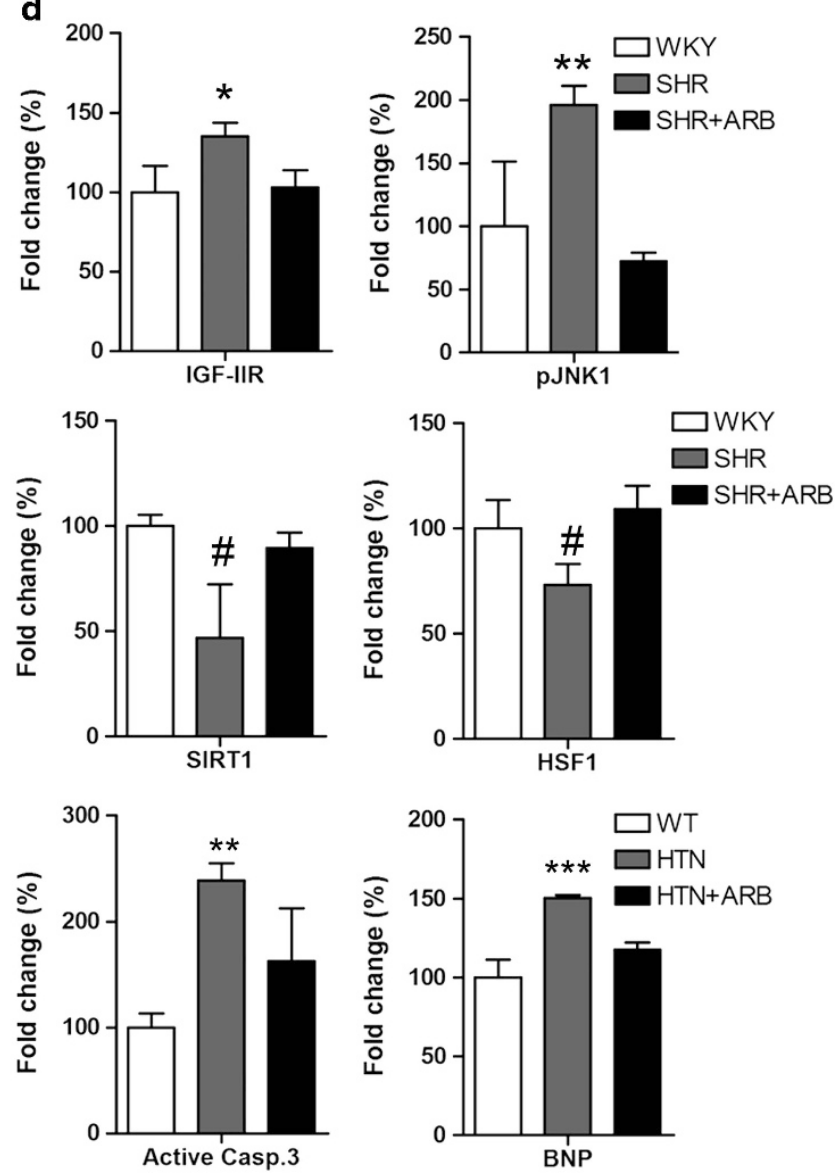

Figure 7 JNK enhanced SIRT1 degradation to activate IGF-IIR- induced hypertrophy and apoptosis in NRVMs and SHR hearts. (a) After treatment with ANG II (100 nM), anisomycin $(50 \mathrm{ng} / \mathrm{ml})$, RSV $(50 \mathrm{mM})$, NAM $(10 \mathrm{mM})$, or anisomycin/NAM for $24 \mathrm{~h}$, H9c2 cell lysates were analyzed by immunoblotting. (b) NRVM primary cells were first transfected with Flag-HSF1 or the HSF1 siRNA (10 nM) for $24 \mathrm{~h}$ and then treated with ANG II (100 nM) and anisomycin $(50 \mathrm{ng} / \mathrm{ml})$, SP600125 $(20 \mu \mathrm{M})$ or NAM (10 mM) for $24 \mathrm{~h}$. ${ }^{*} \mathrm{~A}$ non-specific band. (c) Rat heart tissues were homogenized and extracted for analysis. The expression of IGF-IIR, JNK1/2, SIRT1, HSF1, active caspase-3 and BNP were measured by western blot analysis. (d) The relative expression of IGF-IIR, pJNK1, SIRT1, HSF1, Active Casp.3 and BNP was analyzed by Image J and normalized with the WKY group. ${ }^{*} P<0.05$ represents a significant increase in comparison with untreated control. ${ }^{* *} P<0.01$ represents a significant increase in comparison with untreated control. ${ }^{* \star} P<0.001$ represents a significant increase in comparison with untreated control. ${ }^{\#} P<0.05$ represents a significant decrease in comparison with untreated control 
HSF1 acts as cardioprotector by suppressing IGF-IIR expression

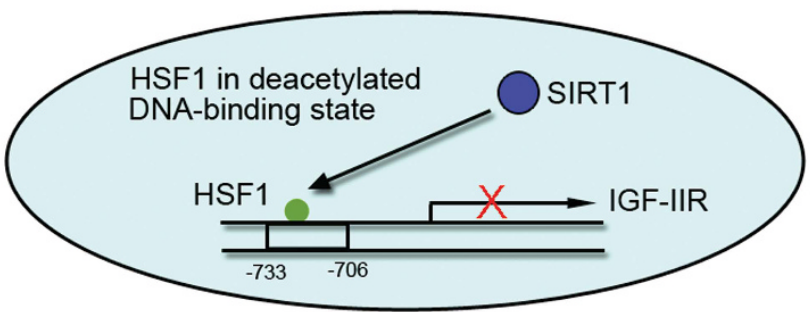

b

Hypentension-induced heart failure

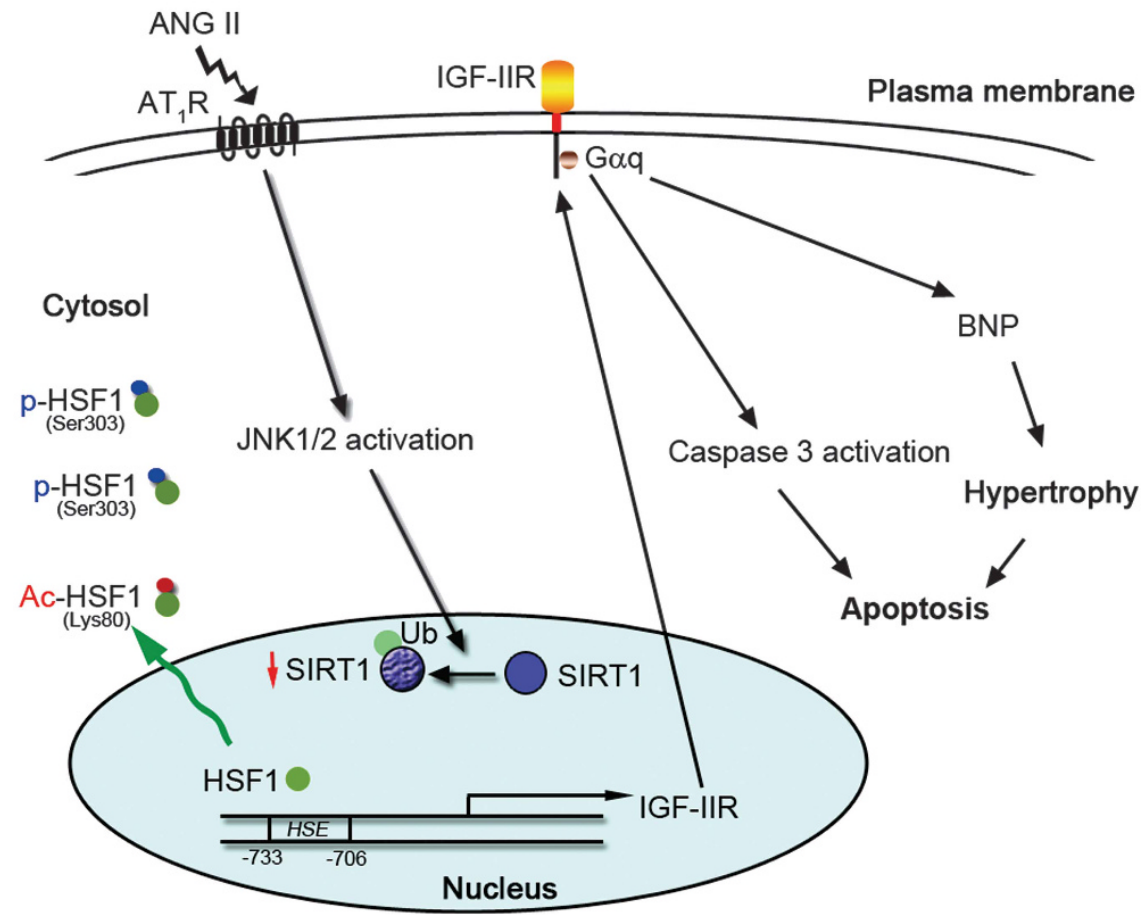

Figure 8 A proposed pathway by which ANG II releases HSF1 from the DNA via the AT 1 R-JNK1/2-SIRT1 pathway, thus upregulating IGF-IIR expression in cardiomyocytes and hypertensive hearts. (a) Under normal conditions, HSF1 acts as repressor to suppress IGF-IIR by binding to its HSE in the IGF-IIR promoter region (nt - 733 to - 706). Therefore, HSF1 protects cardiomyocytes by inhibiting IGF-IIR-induced apoptosis. (b) Once ANG II binds to its receptor $A T_{1} R$, the downstream kinase JNK1/2 is activated to reduce SIRT1 levels via ubiquitin degradation. Without the HSF1 deacetylase SIRT1, the acetylated HSF1 accumulates; at the same time, the levels of the cytosolic inactive HSF1 ${ }^{\text {Ser303 }}$-phosphorylated protein increase. Therefore, HSF1 cannot bind to the IGF-IIR promoter and suppress its expression. IGF-IIR is upregulated and translocates into the surface membrane to sensitize the serum IGF-II, which activates its downstream protein Gaq to induce hypertrophy and apoptosis. Finally, this phenomenon leads to hypertension-induced heart failure

nucleus and binds to the HSE. Recently, HSF1 was recognized as a novel deacetylation target of SIRT1. ${ }^{22,23,38}$ The activation of SIRT1 maintained HSF1 in the deacetylated active state and prolonged its binding to HSEs. Conversely, the downregulation of SIRT1 increased HSF1 acetylation to release HSF1 from HSEs. These results support our observation that ANG II activated JNK and thus caused SIRT1 degradation, leading to an increase in HSF1 acetylation. However, our results do not exclude the possibility that ANG II enhanced HSF1 acetylation by promoting the activity of the acetylase p300. ${ }^{15}$
Previous studies have shown that posttranslational modifications can control the transcriptional activity and DNA-binding ability of HSF1. The phosphorylation of HSF1 at serine 303 and serine 307 has been reported to repress its transcriptional activity, ${ }^{17-19}$ and the acetylation of HSF1 at lysine 80 reduces its binding to the HSE. ${ }^{22,23}$ Moreover, Xavier et al. ${ }^{21}$ found that GSK3 $\beta$-mediated HSF1 phosphorylation regulates both DNA-binding and transcriptional activity, suggesting that HSF1 function is coordinately modulated by posttranslational modifications. Holmberg et al. $^{39}$ also found that phosphorylation by CaMKII at 
serine 230 is essential for HSF1 transcriptional activity, which induces the heat-shock response. However, we did not observe phosphorylation of HSF1 at serine 230 (Supplementary Figures $1 \mathrm{~A}$ and $\mathrm{B}$ ), suggesting that the transcriptional activity of HSF1 was suppressed. Notably, our results show that acetylation and phosphorylation are coordinated to regulate HSF1 function. The sequential order of these HSF1 modifications upon ANG II induction has not yet been determined. Interestingly, Park and $\mathrm{Liu}^{40}$ reported that JNK-mediated HSF1 phosphorylation at serine 444 is independent of its activation. They found that JNK-mediated phosphorylation has a critical role in the stabilization of HSF1 under stress, which explains our observation that the amount of HSF1 is slightly increased and it is phosphorylated into the inactive form after the ANG II challenge in cardiomyocytes (Figures 5 and 6). We suggest that under stress, JNK first initiates HSF1 phosphorylation at serine 444 to stabilize its expression. JNK then degrades SIRT1 to enhance HSF1 acetylation, which is accompanied by HSF1 phosphorylation at serine 303. Eventually, the inactive HSF1 proteins undergo a rapid turnover in SHR heart tissue, resulting in severe heart failure. Our results suggest that the mechanism of HSF1 turnover is an important subject for future investigation.

HSF1 is considered a cardioprotective factor that controls the HSP expression responsible for stress responses in cardiomyocytes. ${ }^{35,41,42}$ We have demonstrated that HSF1 repressed IGF-IIR expression to protect cardiomyocytes under normal conditions. Similarly, recent studies have reported that HSF1 acts as a transcriptional repressor of certain cytokine genes, including TNF- $\alpha$ and IL- $1 \beta$, to prevent LPS-induced inflammation. ${ }^{29,43,44}$ These results support our findings that HSF1 protects cardiomyocytes via transcriptional repression of IGFIIR expression. Our results demonstrate that ANG II represses HSF1 by two mechanisms: (1) phosphorylation of $\mathrm{HSF}_{1}$ Ser303 to inhibit its transcriptional activity and (2) acetylation of HSF ${ }^{\text {Lys } 80}$ to suppress its DNA-binding activity.

Taken together, our observations identify a novel route for IGF-IIR regulation via JNK-SIRT1-HSF1, affecting hypertension-induced heart failure. ANG II binds to its receptor, $A T_{1} R$, which activates the downstream kinase JNK1/2 to degrade SIRT1 via the proteasome. The loss of SIRT1 then increases HSF1 acetylation, resulting in enhanced IGF-IIR expression, which promotes cardiomyocyte hypertrophy and apoptosis. These findings may lead to a therapeutic strategy for regulating hypertension-induced heart failure.

\footnotetext{
Materials and Methods

Experimental animals and oral administration of antihypertension drugs. All animal experiments were performed in accordance with the Guide for the Care and Use of Laboratory Animals (National Institutes of Health Publication No. 85-23, revised 1996) under a protocol approved by the Animal Research Committee of China Medical University, Taichung, Taiwan.

SHRs and normotensive control Wistar-Kyoto (WKY) rats were used in our experiments. The rats were housed at a constant temperature $\left(22^{\circ} \mathrm{C}\right)$ on a $12-\mathrm{h}$ light/dark cycle with food and tap water. The animals were arranged into three groups: WKY rats, SHRs and SHRs treated with irbesartan (SHR/ARB). Each group contained five animals. The ARB drug irbesartan $(40 \mathrm{mg} / \mathrm{kg}$ per day; Merck, Sao Paulo, Brazil) was placed in the drinking water.
}

NRVM primary culture. NRVMs were prepared and cultured using a Neonatal Rat/Mouse Cardiomyocyte Isolation Kit (Cellutron Life Technology, Baltimore, MD, USA). Hearts were dissected from 1- to 3-day-old Sprague-Dawley rats and transferred into a sterile beaker. Each heart was digested and stirred in the beaker at $37^{\circ} \mathrm{C}$ for $12 \mathrm{~min}$. The supernatant was then transferred to a new sterile tube and spun at 1200 r.p.m. for $1 \mathrm{~min}$. The cell pellets were then resuspended in $\mathrm{D} 3$ buffer and preplated for $1 \mathrm{~h}$ by seeding on an uncoated plate at $37^{\circ} \mathrm{C}$ in a $\mathrm{CO}_{2}$ incubator to select the cardiac fibroblasts. The unattached cells were transferred onto plates that were precoated with NS medium (supplemented with $10 \%$ fetal bovine serum). After overnight culture, the NS medium was replaced with a serum-free NW (without serum) medium. The cardiomyocyte cultures were ready for experiments $48 \mathrm{~h}$ after the initial plating.

Expression plasmids and gene construction. Flag-AT $R$ was a gift from Dr. Stephen SG Ferguson (Robarts Research Institute and Department of Physiology and Pharmacology, University of Western Ontario, London, Ontario, Canada). SIRT1-WT, SIRT1-H363Y (dominant-negative mutant) and HSF1 were purchased from Addgene (Cambridge, MA, USA). The IGF-IIR luciferase reporter constructs were generated as described previously. ${ }^{16}$

Cell culture and transient transfection. H9c2 cardiomyoblast cells derived from embryonic BD1X rat heart tissue were obtained from American Type Culture Collection (Manassas, VA, USA) and cultured in Dulbecco's modified essential medium supplemented with $10 \%$ fetal bovine serum, $2 \mathrm{mM}$ glutamine, $100 \mathrm{U} / \mathrm{ml}$ penicillin, $100 \mathrm{mg} / \mathrm{ml}$ streptomycin and $1 \mathrm{mM}$ pyruvate in humidified air $\left(5 \% \mathrm{CO}_{2}\right)$ at $37^{\circ} \mathrm{C}$.

The cells were grown to $80 \%$ confluence on the day of transfection. The plasmids and siRNAs were transfected using the PureFection transfection reagent according to the manufacturer's instructions (System Biosciences, Mountain View, CA, USA). All siRNAs were purchased from Sigma (St. Louis, MO, USA).

Luciferase reporter assay. Briefly, cells were co-transfected with both luciferase IGF-IIR reporter constructs and internal control luciferase plasmids. After transfection and treatment, the cells were assayed for luciferase activity using a Dual-Glo Luciferase Assay System (Promega, Sunnyvale, CA, USA). Plates were read on a Reporter Microplate Luminometer (Turner Biosystems, Sunnyvale, CA, USA). To control for potential variations in transfection or lysis efficiency, the luciferase signals were normalized to the internal control luciferase signal.

Antibodies and reagents. The following antibodies were used in this study: anti-IGF-IIR, anti-SIRT1, anti-JNK1/2, anti-phospho-JNK1/2, anti-ERK1/2, anti-phospho-ERK1/2, anti-PI3K, anti-MEK1/2, anti-phospho-MEK1/2, anti- $\beta$-actin and anti-HDAC1 from Santa Cruz Biotechnology (Santa Cruz, CA, USA); anti-Flag and anti-acetyl-lysine from Abcam (Cambridge, UK); anti-HSF1 and antiphospho-PI3K from Cell Signaling Technology (Danvers, MA, USA). All the secondary antibodies (anti-rabbit, mouse and goat, HRP-conjugated) were purchased from Santa Cruz Biotechnology. All reagents were purchased from Sigma.

Western blot analysis and immunoprecipitation. A total of $30 \mu \mathrm{g}$ of total lysates or $10 \mu \mathrm{g}$ of subcellular fractions was separated using $6-12 \%$ SDS-polyacrylamide gel electrophoresis and then transferred to PVDF membranes (GE Healthcare, Amersham, UK). The membranes were blocked using $5 \%$ non-fat milk and blotted with specific antibodies overnight at $4{ }^{\circ} \mathrm{C}$. Then, the protein signals were measured using horseradish peroxidase-conjugated secondary antibodies (1:10000; GE Healthcare) and Immobilon Western Chemiluminescent HRP Substrate (Millipore, Billerica, MA, USA). Densitometric analysis of the immunoblots was performed using the Alphalmager2200 digital imaging system (Digital Imaging System, Commerce, CA, USA). The digital images were processed in Adobe Photoshop 7.0. Each blot was stripped using Restore Western Blot Stripping Buffer (Pierce, Rockford, IL, USA) and incubated with other antibodies. The results were analyzed and quantified by the Image $J$ software (NIH, Bethesda, MD, USA).

Immunoprecipitations were performed from $\mathrm{H} 9 \mathrm{c2}$ cell lysates using the PureProteome Protein G Magnetic Bead System (Millipore) according to the manufacturer's instructions. A total of $300 \mu \mathrm{g}$ of cell lysate was prepared. The lysate was combined and allowed to interact with $2 \mu \mathrm{g}$ of a specific primary antibody, and the mixture was incubated on a rotator at $4^{\circ} \mathrm{C}$ overnight. Immunoprecipitated proteins were eluted from magnetic beads at $95^{\circ} \mathrm{C}$ for $5 \mathrm{~min}$ and separated by SDS-PAGE. The proteins were transferred to a PVDF membrane and probed with specific antibodies. 
RNA extraction and RT-PCR. Total RNA was extracted using the Directzol RNA MiniPrep Kit (Zymo Research Corporation, Irvine, CA, USA) according the manufacturer's instructions. Briefly, $1 \mu \mathrm{g}$ of total RNA was incubated with $0.5 \mu \mathrm{g}$ of oligo dT (MD. Bio., Taipei, Taiwan) at $70^{\circ} \mathrm{C}$ for $15 \mathrm{~min}$. Then, the RNA was mixed with buffer containing $0.25 \mathrm{mM}$ dNTPs (MD. Bio.), $20 \mathrm{U}$ of RNasin I Plus RNase Inhibitor (Promega, Madison, WI, USA) and $20 \mathrm{U}$ of M-MLV Reverse Transcriptase (Promega) and incubated at $42^{\circ} \mathrm{C}$ for $90 \mathrm{~min}$ for cDNA synthesis. This mixture was then used for specific cDNA amplification in a GeneAmp PCR system 2400 (Perkin-Elmer, Waltham, MA, USA).

ChIP assay. A ChIP assay was performed using lysates from H9c2 cells that were treated with ANG II. The assay was performed using EZ ChIP Chromatin (Millipore, Temecula, CA, USA) according to the manufacturer's instructions. The cells were washed in PBS three times and incubated for 10 min with $1 \%$ formaldehyde. After quenching with $0.1 \mathrm{M}$ glycine, the crosslinked material was sonicated into chromatin fragments with an average length of 500-800 bp. The chromatin was kept at $-80^{\circ} \mathrm{C}$. The chromatin solution $(100 \mu \mathrm{l}$ of chromatin sample and $900 \mu$ l of dilution buffer) was precleared by adding Protein $G$ agarose for $2 \mathrm{~h}$ at $4{ }^{\circ} \mathrm{C}$, and immunoprecipitation was then performed with Protein $\mathrm{G}$ agarose and $1-10 \mu \mathrm{g}$ of the indicated antibodies overnight at $4^{\circ} \mathrm{C}$ on a rotating wheel. The immunoprecipitated material was washed five times with cold washing buffer. The crosslinks were reversed by incubating the samples with $8 \mu \mathrm{l}$ of $5 \mathrm{M}$ $\mathrm{NaCl}$ for $5 \mathrm{~h}$ at $65^{\circ} \mathrm{C}$, and $10 \mu \mathrm{g}$ of RNase A was added to eliminate the RNA. The recovered material was treated with proteinase $\mathrm{K}$, placed in spin columns and precipitated. The pellets were resuspended in $50 \mu \mathrm{l}$ of double-distilled water and analyzed using PCR.

Indirect immunofluorescence and confocal microscopy. Cells were fixed with $4 \%$ paraformaldehyde for $15 \mathrm{~min}$ at room temperature and permeabilized with $0.1 \%$ Triton $\mathrm{X}-100$ for $15 \mathrm{~min}$ at room temperature before staining with a specific antibody. Then, the cells were washed and stained with Alexa 546 rabbit anti-mouse lgG secondary antibodies (Invitrogen, Carlsbad, CA USA). Images were captured using a Leica SP2 Confocal Spectral Microscope (Buffalo Grove, IL, USA). The images were processed using Adobe Photoshop.

Measurement of surface IGF-IIR expression. Cells were seeded into 12-well plates on the day before treatment with siRNAs or drugs. After treatment, the cells were washed using PBS and fixed with $4 \%$ paraformaldehyde for $15 \mathrm{~min}$ at room temperature. Then, the cells were blocked with $5 \%$ goat serum and incubated with a mouse anti-IGF-IIR antibody (ab2733; Abcam) overnight at $4{ }^{\circ} \mathrm{C}$ After staining with the primary antibody, the cells were incubated with a rabbit antimouse HRP-conjugated antibody for $1.5 \mathrm{~h}$ at room temperature. Finally, the cells were washed and incubated with the HRP substrate (1-step ultra TMB solution; Pierce) for $30 \mathrm{~min}$. The reaction was stopped using $1 \mathrm{M}$ sulfuric acid. The sample was measured at $550 \mathrm{~mm}$.

Statistical analysis. All experiments were performed at least three times. Statistical analysis was performed using the GraphPad Prism5 statistical software (GraphPad, San Diego, CA, USA). Statistical significance was set at $P<0.05$. Multiple comparisons of the data were analyzed by the ANOVA assay. All results were quantified by Image $\mathrm{J}(\mathrm{NIH})$ and processed using Adobe Photoshop.

\section{Conflict of Interest}

The authors declare no conflict of interest.

Acknowledgements. The experiments and data analysis were performed using the Medical Research Core Facilities Center, Office of Research and Development at China Medical University, Taichung, Taiwan. This study is supported by the Taiwan Department of Health Clinical Trial and Research Center of Excellence (DOH102-TD-B-111-004).

1. George AJ, Thomas WG, Hannan RD. The renin-angiotensin system and cancer: old dog, new tricks. Nat Rev Cancer 2010; 10: 745-759.

2. Herichova I, Szantoova K. Renin-angiotensin system: upgrade of recent knowledge and perspectives. Endocr Regul 2013; 47: 39-52.
3. Mehta PK, Griendling KK. Angiotensin II cell signaling: physiological and pathological effects in the cardiovascular system. Am J Physiol Cell Physiol 2007; 292: C82-C97.

4. Hunyady L, Catt KJ. Pleiotropic AT1 receptor signaling pathways mediating physiological and pathogenic actions of angiotensin II. Mol Endocrinol 2006; 20: 953-970.

5. Braulke T. Type-2 IGF receptor: a multi-ligand binding protein. Horm Metab Res 1999; 31(2-3): 242-246.

6. Hawkes C, Kar S. The insulin-like growth factor-II/mannose-6-phosphate receptor: structure, distribution and function in the central nervous system. Brain Res Brain Res Rev 2004; 44: 117-140.

7. Lau MM, Stewart CE, Liu Z, Bhatt H, Rotwein P, Stewart CL. Loss of the imprinted IGF2/cation-independent mannose 6-phosphate receptor results in fetal overgrowth and perinatal lethality. Genes Dev 1994; 8: 2953-2963.

8. Wylie AA, Pulford DJ, McVie-Wylie AJ, Waterland RA, Evans HK, Chen YT et al. Tissuespecific inactivation of murine M6P/IGF2R. Am J Pathol 2003; 162: 321-328.

9. Kuo WW, Liu CJ, Chen LM, Wu CH, Chu CH, Liu JY et al. Cardiomyoblast apoptosis induced by insulin-like growth factor (IGF)-I resistance is IGF-II dependent and synergistically enhanced by angiotensin II. Apoptosis 2006; 11: 1075-1089.

10. Lee SD, Chu CH, Huang EJ, Lu MC, Liu JY, Liu CJ et al. Roles of insulin-like growth factor II in cardiomyoblast apoptosis and in hypertensive rat heart with abdominal aorta ligation. Am J Physiol Endocrinol Metab 2006; 291: E306-E314.

11. Chu CH, Tzang BS, Chen LM, Kuo CH, Cheng YC, Chen LY et al. IGF-II/mannose-6phosphate receptor signaling induced cell hypertrophy and atrial natriuretic peptide/BNP expression via Galphaq interaction and protein kinase C-alpha/CaMKII activation in H9c2 cardiomyoblast cells. J Endocrinol 2008; 197: 381-390.

12. Chu CH, Huang CY, Lu MC, Lin JA, Tsai FJ, Tsai CH et al. Enhancement of AG1024induced $\mathrm{H} 9 \mathrm{c} 2$ cardiomyoblast cell apoptosis via the interaction of IGF2R with Galpha proteins and its downstream PKA and PLC-beta modulators by IGF-II. Chin J Physio/ 2009; 52: $31-37$.

13. Chu CH, Tzang BS, Chen LM, Liu CJ, Tsai FJ, Tsai $\mathrm{CH}$ et al. Activation of insulin-like growth factor II receptor induces mitochondrial-dependent apoptosis through $\mathrm{G}($ alpha)q and downstream calcineurin signaling in myocardial cells. Endocrinology 2009; 150: 2723-2731.

14. Chu CH, Lo JF, Hu WS, Lu RB, Chang MH, Tsai FJ et al. Histone acetylation is essential for ANG-II-induced IGF-IIR gene expression in H9c2 cardiomyoblast cells and pathologically hypertensive rat heart. J Cell Physiol 2012; 227: 259-268.

15. Anckar J, Sistonen L. Regulation of HSF1 function in the heat stress response: implications in aging and disease. Annu Rev Biochem 2011; 80: 1089-1115.

16. Neef DW, Jaeger AM, Thiele DJ. Heat shock transcription factor 1 as a therapeutic target in neurodegenerative diseases. Nat Rev Drug Discov 2011; 10: 930-944.

17. Chu B, Soncin F, Price BD, Stevenson MA, Calderwood SK. Sequential phosphorylation by mitogen-activated protein kinase and glycogen synthase kinase 3 represses transcriptional activation by heat shock factor-1. J Biol Chem 1996; 271: 30847-30857.

18. Knauf U, Newton EM, Kyriakis J, Kingston RE. Repression of human heat shock factor 1 activity at control temperature by phosphorylation. Genes Dev 1996; 10: 2782-2793.

19. Kline MP, Morimoto RI. Repression of the heat shock factor 1 transcriptional activation domain is modulated by constitutive phosphorylation. Mol Cell Biol 1997; 17: 2107-2115.

20. Chu B, Zhong R, Soncin F, Stevenson MA, Calderwood SK. Transcriptional activity of heat shock factor 1 at 37 degrees $C$ is repressed through phosphorylation on two distinct serine residues by glycogen synthase kinase 3 and protein kinases Calpha and Czeta. J Biol Chem 1998; 273: 18640-18646.

21. Xavier IJ, Mercier PA, McLoughlin CM, Ali A, Woodgett JR, Ovsenek N. Glycogen synthase kinase 3beta negatively regulates both DNA-binding and transcriptional activities of heat shock factor 1. J Biol Chem 2000; 275: 29147-29152.

22. Westerheide SD, Anckar J, Stevens Jr SM, Sistonen L, Morimoto RI. Stress-inducible regulation of heat shock factor 1 by the deacetylase SIRT1. Science 2009; 323: 1063-1066.

23. Raynes R, Pombier KM, Nguyen K, Brunquell J, Mendez JE, Westerheide SD. The SIRT1 modulators AROS and DBC1 regulate HSF1 activity and the heat shock response. PLOS One 2013; 8: e54364

24. Ford J, Ahmed S, Allison S, Jiang M, Milner J. JNK2-dependent regulation of SIRT1 protein stability. Cell Cycle 2008; 7: 3091-3097.

25. Gao Z, Zhang J, Kheterpal I, Kennedy N, Davis RJ, Ye J. Sirtuin 1 (SIRT1) protein degradation in response to persistent c-Jun N-terminal kinase 1 (JNK1) activation contributes to hepatic steatosis in obesity. J Biol Chem 2011; 286: 22227-22234.

26. Wang ZQ, Fung MR, Barlow DP, Wagner EF. Regulation of embryonic growth and lysosomal targeting by the imprinted Igf2/Mpr gene. Nature 1994; 372: 464-467.

27. Ludwig T, Eggenschwiler J, Fisher P, D'Ercole AJ, Davenport ML, Efstratiadis A. Mouse mutants lacking the type 2 IGF receptor (IGF2R) are rescued from perinatal lethality in Igf2 and Igf1r null backgrounds. Dev Biol 1996; 177: 517-535.

28. Huang CY, Lee SD. Possible pathophysiology of heart failure in obesity: Cardiac apoptosis. Biomedicine 2012; 2: 36-40

29. Chen RJ, Wu HC, Chang MH, Lai CH, Tien YC, Hwang JM et al. Leu27IGF2 plays an opposite role to IGF1 to induce $\mathrm{H} 9 \mathrm{c} 2$ cardiomyoblast cell apoptosis via Galphaq signaling. J Mol Endocrinol 2009; 43: 221-230. 
30. Planavila A, Dominguez E, Navarro M, Vinciguerra M, Iglesias R, Giralt M et al. Dilated cardiomyopathy and mitochondrial dysfunction in Sirt1-deficient mice: a role for Sirt1-Mef2 in adult heart. $J$ Mol Cell Cardiol 2012; 53: 521-531.

31. Biala A, Tauriainen E, Siltanen A, Shi J, Merasto S, Louhelainen M et al. Resveratrol induces mitochondrial biogenesis and ameliorates Ang II-induced cardiac remodeling in transgenic rats harboring human renin and angiotensinogen genes. Blood Press 2010; 19 : 196-205.

32. Li L, Gao P, Zhang H, Chen H, Zheng W, Lv X et al. SIRT1 inhibits angiotensin II-induced vascular smooth muscle cell hypertrophy. Acta Biochim Biophys Sin (Shanghai) 2011; 43: 103-109.

33. Becatti M, Taddei N, Cecchi C, Nassi N, Nassi PA, Fiorillo C. SIRT1 modulates MAPK pathways in ischemic-reperfused cardiomyocytes. Cell Mol Life Sci 2012; 69: 2245-2260

34. Kuno A, Hori YS, Hosoda R, Tanno M, Miura T, Shimamoto K et al. Resveratrol improves cardiomyopathy in dystrophin-deficient mice through SIRT1 protein-mediated modulation of p300 protein. J Biol Chem 2013; 288: 5963-5972.

35. Yin C, Xi L, Wang X, Eapen M, Kukreja RC. Silencing heat shock factor 1 by small interfering RNA abrogates heat shock-induced cardioprotection against ischemiareperfusion injury in mice. J Mol Cell Cardiol 2005; 39: 681-689.

36. Zou Y, Li J, Ma H, Jiang H, Yuan J, Gong H et al. Heat shock transcription factor 1 protects heart after pressure overload through promoting myocardial angiogenesis in male mice. J Mol Cell Cardiol 2011; 51: 821-829.
37. Wu L, Hu C, Huang M, Jiang M, Lu L, Tang J. Heat shock transcription factor 1 attenuates TNFalpha-induced cardiomyocyte death through suppression of NFkappaB pathway. Gene 2013; 527: 89-94.

38. Ghemrawi R, Pooya S, Lorentz S, Gauchotte G, Arnold C, Gueant JL et al. Decreased vitamin B12 availability induces ER stress through impaired SIRT1-deacetylation of HSF1. Cell Death Dis 2013; 4: e553.

39. Holmberg $\mathrm{Cl}$, Hietakangas V, Mikhailov A, Rantanen JO, Kallio M, Meinander A et al. Phosphorylation of serine 230 promotes inducible transcriptional activity of heat shock factor 1. EMBO J 2001; 20: 3800-3810.

40. Park J, Liu AY. JNK phosphorylates the HSF1 transcriptional activation domain: role of JNK in the regulation of the heat shock response. J Cell Biochem 2001; 82: 326-338.

41. Griffin TM, Valdez TV, Mestril R. Radicicol activates heat shock protein expression and cardioprotection in neonatal rat cardiomyocytes. Am J Physiol Heart Circ Physiol 2004; 287: $\mathrm{H} 1081-\mathrm{H} 1088$.

42. Peng W, Zhang Y, Zheng M, Cheng H, Zhu W, Cao CM et al. Cardioprotection by CaMKIIdeltaB is mediated by phosphorylation of heat shock factor 1 and subsequent expression of inducible heat shock protein 70. Circ Res 2010; 106: 102-110.

43. Xie Y, Chen C, Stevenson MA, Auron PE, Calderwood SK. Heat shock factor 1 represses transcription of the IL-1beta gene through physical interaction with the nuclear factor of interleukin 6. J Biol Chem 2002; 277: 11802-11810.

44. Singh IS, He JR, Hester L, Fenton MJ, Hasday JD. Bacterial endotoxin modifies heat shock factor-1 activity in RAW 264.7 cells: implications for TNF-alpha regulation during exposure to febrile range temperatures. J Endotoxin Res 2004; 10: 175-184.

Supplementary Information accompanies this paper on Cell Death and Differentiation website (http://www.nature.com/cdd) 